\title{
NOTE ON THE SETTING OF A MERCURY SURFACE TO A REQUIRED HEIGHT
}

\author{
By M. H. Stillman.
}

The present brief article has to do with an improvement in the well-known method of adjusting a mercury surface to a given height by bringing it just into contact with the lower end of a pointer of ivory or other material which projects from above downward toward the surface of the mercury. This method is commonly made use of in certain types of mercurial barometers.

In setting the mercury surface in the cistern of a barometer it is usual to raise the surface of the mercury to the required height in order that the barometer may always be read with a rising meniscus, so as to reduce to a minimum the irregularity due to capillarity and to the sticking of the mercury to the sides of the tube. The mercury surface is therefore raised until the tip of the pointer and its image in the mercury seem to coincide or until a tiny dimple is formed in the mercury by the tip of the pointer.

The improvement referred to above is an artifice by means of which a smaller dimple can be detected, and consists simply of a scale of alternate parallel white and black lines, each about 0.5 mm wide, placed back of the pointer in such a way that the observer sees the image of this scale in the mercury surface at the point where the dimple is to be formed. When the pointer is not touching the mercury the surface of the latter will be practically plane, and the lines of the image of the scale will appear parallel; but, as soon as a very small dimple is formed by the 
contact of the pointer and mercury, the fact is made evident by a distortion of the lines of the image of the scale. The advantages apparent in the use of this auxiliary scale are the increased ease and accuracy with which the mercury surface may be set by using only ordinary care, and the consequent reduced eye strain when a large number of readings are made.

In Figs. I and 2 are represented the appearances of the mercury surface in the cistern of a barometer equipped with the above-mentioned auxiliary scale, before and after setting, respectively. It will be noted that while in Fig. I, with the mercury below the end of the pointer, the lines of the image are practically parallel below the pointer, in Fig. 2 the images of the lines of the scale are distorted at the end of the pointer, indicating contact between the latter and the mercury. In Fig. 2, in order that the photograph might show the distortion of the lines more plainly, the mercury was set higher relative to the pointer than would be the case in an actual reading of the barometer.

In order to determine the accuracy with which settings of a pointer to the mercury surface may be repeated by this method, the experiment described below was made.

Fig. 3 is from a photograph of the apparatus used in the determination. A steel pointer (P) was attached to the foot of the movable scale (S) of a Zeiss vertical comparator and a slowmotion screw (A) adjusted to bear upon the upper end of this scale, so that the latter with the attached pointer could be slowly and steadily raised from or lowered to the mercury surface (M) below. B B are stretched rubber bands attached to the scale to maintain it in contact with the height-adjusting mechanism (A). The parallel-line scale (L) was placed behind the pointer, as shown, so that the desired reflection could be obtained in the mercury surface. The vertical scale (S) was read by means of the comparator microscope, which was provided with a micrometer eyepiece.

The steel pointer was a wire of about I mm diameter, with its lower end rounded to approximately a hemisphere of the diameter of the wire. Thus, the pointer was very similar in shape to those commonly used in mercury barometers, although, of course, not of the same material 


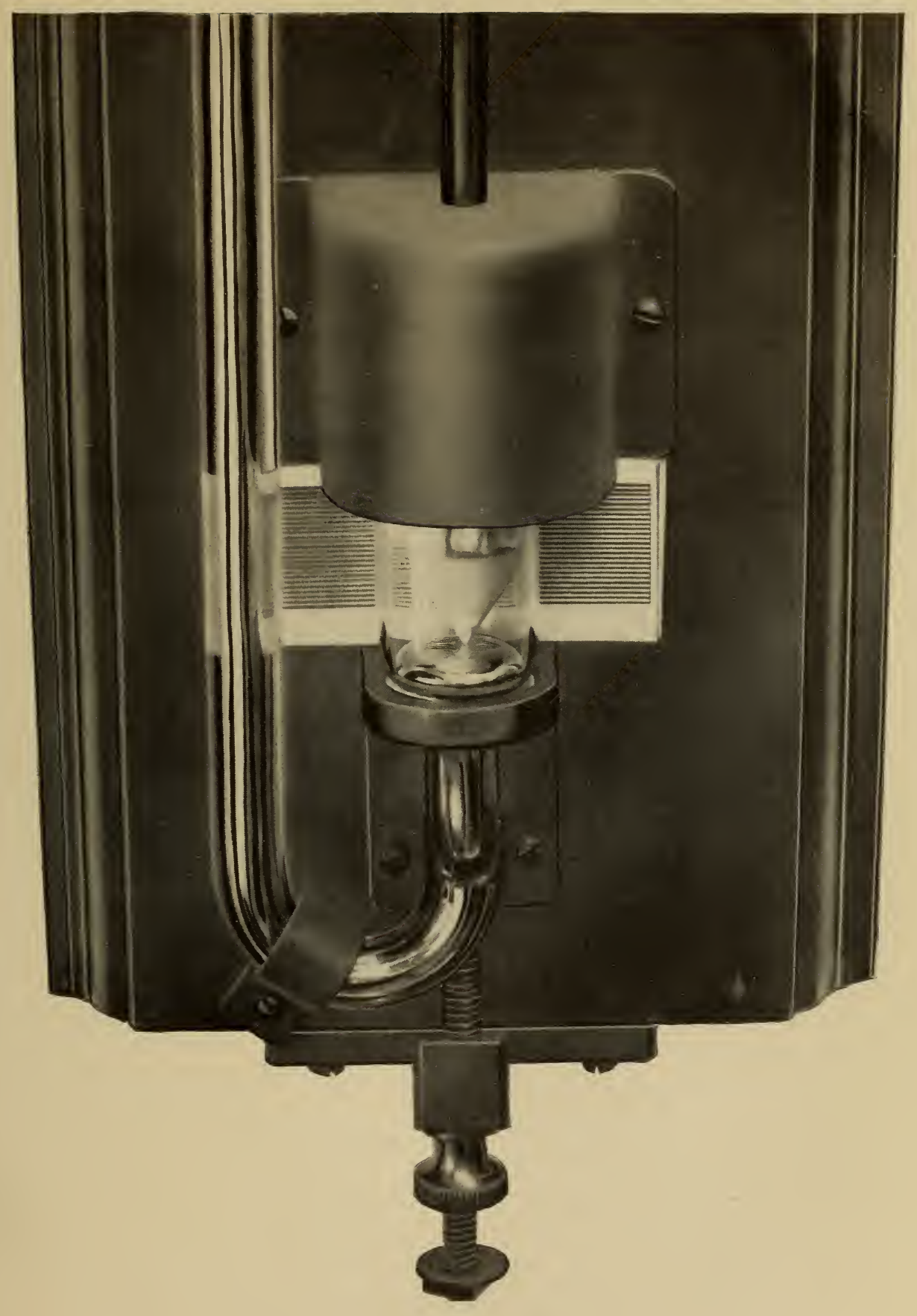

Fig. 2 

The method of procedure in making the measurements was to lower the pointer slowly until a distortion of the parallel lines of the image was visible and then to read the scale by means of the micrometer microscope. No auxiliary device was used to aid the eye in detecting distortion of the lines.

Below is given a sample set of five consecutive readings made in the manner described above.

\begin{tabular}{c|c|c}
\hline Number of observation & $\begin{array}{c}\text { Reading at dimple } \\
\text { contact }\end{array}$ & $\begin{array}{c}\text { Deviation from } \\
\text { meen }\end{array}$ \\
\hline & mm & mm \\
& 46.8242 & 0.0003 \\
.8242 & .0003 \\
.0000 \\
.8239 & .0007 \\
.0003 \\
\hline
\end{tabular}

This table indicates the rather surprising fact that all of the successive independent settings of the pointer and micrometer agreed with the mean setting well within $0.00 \mathrm{rm}$ and that the average deviation from this mean was only $0.0003 \mathrm{~mm}$. It was found that even better agreement of readings than the above recorded could be obtained when the illumination and the condition of the observer's eyes were more favorable.

The accuracy with which settings could ordinarily be reproduced, using the method of coincidence of point and image, was also investigated and found to be less than by using the improved method. Thus, while the average deviation of the settings from the mean in the case of the improved method was $0.0003 \mathrm{~mm}$, the other method, under very favorable conditions, showed an average deviation of $0.0026 \mathrm{~mm}$ in a similar group of five settings.

It is of course to be expected that the height of the pointer (relative to the mercury surface), when set by the improved method, will be slightly less than when set by noting the apparent coincidence of the end of the pointer and its image or by noting when light can no longer be seen between the pointer and the mercury surface; in the settings by the two last-mentioned methods, the end of the pointer is probably slightly above the 
surface, while in the setting by the first-mentioned method (the improved method) the end of the pointer is necessarily a finite distance below the surface in order to produce the dimple. Determinations made with the apparatus described above indicated that this difference in the settings was ordinarily from $0.012 \mathrm{~mm}$ to $0.018 \mathrm{~mm}$ and that this improved method gives a setting between that of the ordinary dimple method and the method depending upon the apparent coincidence of the end of the pointer and its image. The above-mentioned differences are negligible in ordinary barometry but should be considered in extremely precise work if a barometer, the corrections of which are known to a high degree of accuracy, is used. The improved method should be especially valuable where it is practical to use it in determining the difference in height of two mercury surfaces; for in this case the constant errors of the settings cancel, thus allowing the full advantage of the sensitiveness of the method to be realized.

Unfortunately, not all barometers in which the lower mercury surface is set to the end of a pointer have been designed with sufficient space between the mercury surface and the top of the cistern to allow the improved method to be used with maximum advantage. There are, however, few barometers of this type with which considerable advantage both in convenience and precision of setting can not be gained either by placing the scale behind the cistern of the barometer or within it.

WASHINGTON, September 2O, I9I3. 


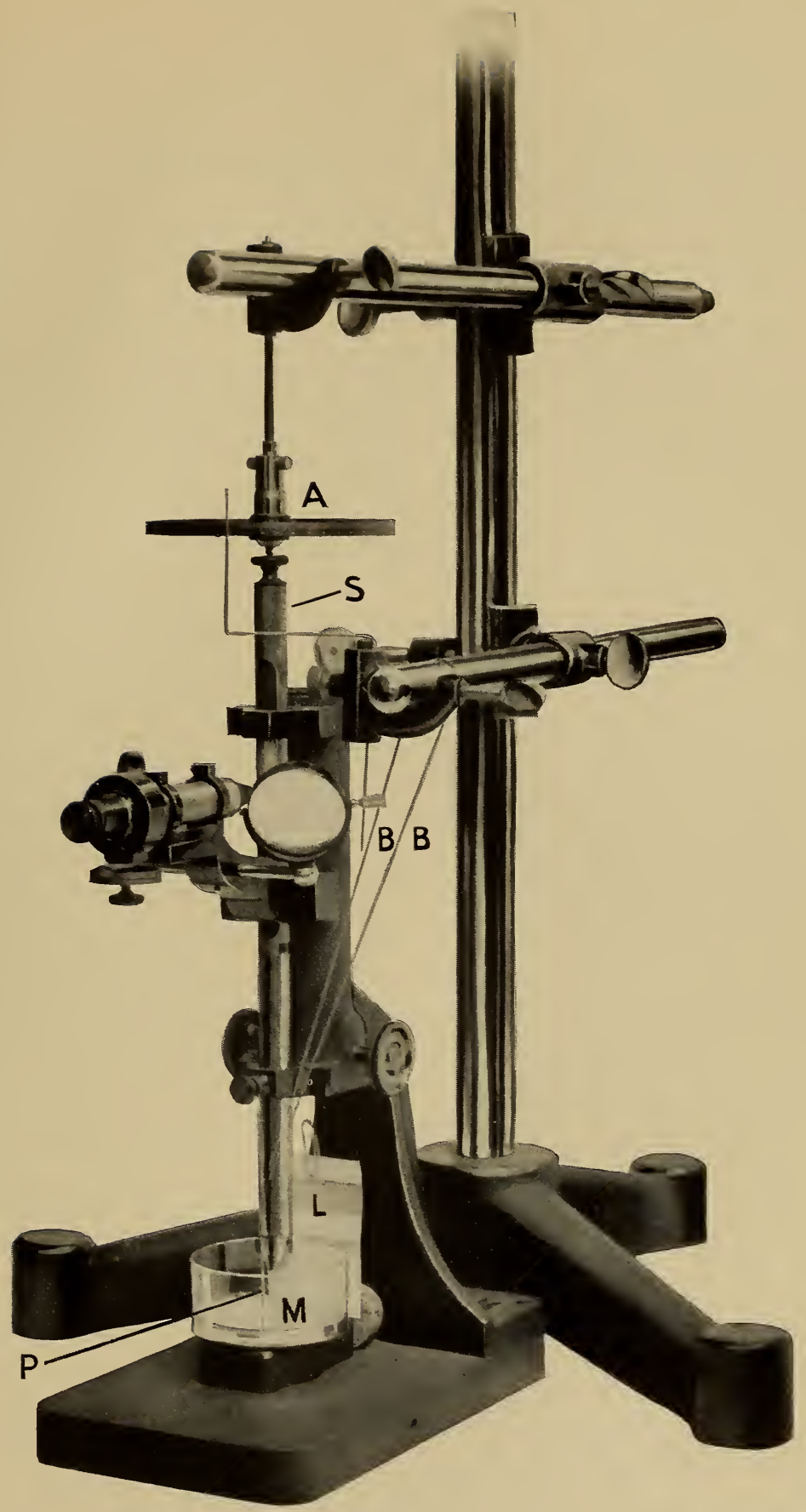

Fig. 3 



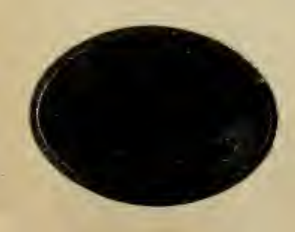


